\title{
ENEMIGOS NATURALES DE LA MOSCA BLANCA Aleurodicus dispersus RUSSELL (HOMOPTERA: ALEYRODIDAE): PARASITOIDES Y DEPREDADORES
}

\author{
Belga Blanco-Metzler ${ }^{1}$, Sergio Laprade ${ }^{2}$
}

\begin{abstract}
RESUMEN
Enemigos naturales de la mosca blanca Aleurodicus dispersus Russell (Homoptera: Aleyrodidae): parasitoides y depredadores. Se estudió la composición de parasitoides y depredadores de la mosca blanca en espiral Aleurodicus dispersus así como la abundancia relativa de las primeras diez fincas productoras de banano, durante 1997 y 1998 en Matina, Limón, Costa Rica. Se recuperaron cuatro especies de parasitoides de ninfas de mosca blanca Encarsiella noyesi Hayat, E. aleurodici (Girault), Encarsiella probo nsp. y Encarsia guadeloupae Viggiani. Los depredadores de ninfas y adultos de mosca blanca más comunes fueron dos especies de ácaros pertenecientes a la familia Phytoseiidae, Nephaspis sp. (Coleoptera: Coccinelidae), Scymnus sp. (Coleoptera: Coccinelidae), y tres especies de arañas: Plesiometa argyra (Araneida), Gasteracantha cancriformis (Araneida), y Chrysso sp. (Araneida). El porcentaje de parasitismo difirió significativamente entre fincas $y$ fue mayor en AGRODISA $>$ SEMDEL $>$ Anabel.
\end{abstract}

\begin{abstract}
Natural enemies of the spiralling whitefiy, Aleurodicus dispersus Russell (Homoptera: Aleyrodidae): Parasitoids y depredators. Composition of species of predators and parasitoid, as well as parasitoid abundance between banana farms associated with the spiralling whitefly, Aleurodicus dispersus Russell, were studied during 1997 and 1998 in Matina, Limltn, Costa Rica. Four parasitoids, Encarsiella noyesi Hayat, E. aleurodici (Girault), Encarsiella probo nsp. and Encarsia guadeloupae Viggiani were recovered from whitefly nymphs. Two species of mite belonging to Phytoseiidae family, Nephaspis sp. (Coleoptera: Coccinelidae), Scymnus sp. (Coleoptera: Coccinelidae), and three species of spiders: Plesiometa argyra (Araneida), Gasteracantha cancriformis (Araneida), and Chrysso sp. (Araneida), were the most common predators of whitefly aduIt and nymphs. Percentage of parasitism between farms was found to differ significantly being highcst in AGRODISA $>$ SEMDEL $>$ Anabel.
\end{abstract}

\section{INTRODUCCIÓN}

La mosca blanca en espiral Aleurodicus dispersus Russell (Homoptera: Aleyrodidae) es una plaga tropical con gran cantidad de hospederos, donde se informan más de 100 especies de plantas, hortalizas, árboles frutales, palmeras, ornamentales, árboles de sombra y varias malezas (Waterhouse y Norris, 1989; Blanco-Metzler y Laprade, 1998).

Este insecto presenta características que 10 convierten en una plaga potencial para numerosos cultivos, como son su capacidad de alimentación y reproducción en muchos hospederos, su capacidad de reproducción sin necesidad de que las hembras sean fecundadas (partenogéne- sis arrenotóquica) y de presentar incrementos poblacionales muy grandes en corto tiempo (Lambkin, 1996a; Blanco-Metzler y Laprade, 1998). La mosca blanca pasa por cuatro estadíos ninfales entre los estados de huevo y adulto, donde todos los estadíos ninfales y el adulto se alimentan de la savia de las hojas. La mosca blanca produce secreciones azucaradas las cuales sirven de sustrato al crecimiento de fumagina (Capnodium sp. ?), 10 que a su vez reduce la fotosíntesis, afectando la producción de banano (Laprade y Blanco-Metzler, en prensa).

En 1995 se detectó la presencia de la mosca blanca en dos fincas de Matina, provincia de Limón, en poblaciones de hasta 7000 ninfas por hoja de banano. Se utilizaron como estrategias de combate, insecticidas,

\footnotetext{
1 Centro de Investigación en Protección de Cultivos-Estación Experimental Fabio Baudrit Moreno, Universidad de Costa Rica, Facultad de Agronomía.e-mail: hblanco@cariari.ucr.ac.cr

2 Dirección de Investigaciones, CORBANA. Apdo 6504-1000 San José, Costa Rica.
} 
reguladores de crecimiento y compuestos naturales y el control biológico (Laprade y Cerdas, 1997).

En la finca Turquesa Dorada se introdujeron 522 pupas de Encarsia sp. (Homoptera: Aphelinidae), parasitoide de A. dispersus, provenientes de la Isla Thursday en Torres Strait, (Lambkin 1996b). Estudios posteriores permitieron identifica otro parasitoide Encarsiella spp., del cual se desconocía su presencia, a pesar de ser un parasitoide nativo.

El presente estudio tuvo como objetivos: hacer un inventario de los enemigos naturales de $A$. dispersus en las plantaciones bananeras, determinar si el parasitoide Encarsia sp. se había establecido en la zona y cuantificar la abundancia poblacional de los parasitoides en diez fincas bananeras.

\section{MATERIALES Y MÉTODOS}

\section{Identificación de los enemigos naturales}

El estudio se llevó a cabo entre mayo de 1997 a febrero de 1998, en las fincas Anabel, PROBANA, Hacienda Río Palacios, Rinos, AGRODISA y Turquesa Dorada ubicadas en el cantón de Matina, Limón, Costa Rica, a 20 m.s.n.m. y en el laboratorio del Centro de Investigaciones de CORBANA en 28 Millas, en el cantón de Matina, provincia de Limón. La precipitación promedio fue de $255,7 \mathrm{~mm}$, la temperatura promedio de 24 'c y la humedad relativa del $83,3 \%$.

Durante los diez meses de trabajo, se realizó una búsqueda de aquellos enemigos naturales que pudieran estar incidiendo en la regulación de la población de $A$. dispersus. En cada finca, en cuatro ocasiones, se recolectaron mlJestras de hojas de banano, malezas y plantas ornamentales también hospederas de la mosca blanca, que presentaban ninfas parasitadas y se llevaron al laboratorio. Se consideró una ninfa parasitada, como aquella de tercer o cuarto estadío, que al remover con un pincel fino la cera que ella misma produce, se observara un color café oscuro y que no presentara un orificio de emergencia del parasitoide adulto. Cada especie vegetal se introdujo en recipientes de plástico de medio galón hasta la emergencia de los parasitoides. Los recipientes tenían un agujero redondo en la tapa (10 cm de diámetro) forrados con una malla fina de nylon cristal. Además, se recolectaron enemigos naturales en el campo, principalmente arañas y coleópteros, luego se introdujeron en frascos con alcohol al 70\% para su identificación.

Debido a que se observó que de las numerosas espirales con oviposiciones frescas, sólo unos pocos hue- vos pasaban al estado de ninfa y que de éstas sólo unas pocas completaban su ciclo de vida, se inició una serie de observaciones para determinar el agente causal. Se recolectaron muestras de banano y de otros hospederos donde la espiral tuviera aspecto borroso y se llevaron al laboratorio para observarlas bajo el estereoscopio.

Se recolectaron muestras de ninfas parasitadas con un hongo y se llevaron al laboratorio de patología de la Universidad de Costa Rica en Turrialba para el aislamiento, reproducción e identificación.

\section{Abundancia de parasitoides}

En cada finca, en doce ocasiones, se seleccionaron al azar cinco plantas de banano. Durante las tres primeras evaluaciones se muestrearon tres plantas por finca, posteriormente fue aumentado a cinco plantas por finca. El muestreo se realizó en la parte abaxial de la mitad de la lámina foliar de la octava hoja, según la metodología utilizada en el muestreo de la sigatoka negra. Se registró el número de ninfas parasitadas según la metodología descrita.

El diseño utilizado fue un irrestricto al azar con diez fincas y cinco repeticiones. La separación de medias se realizó por medio de la prueba de Duncan. Debido a la frecuente aparición de ceros en los muestreos, se transformaron los daros por la fórmula $\sqrt{ } X+0,5$.

\section{RESULTADOS Y DISCUSIÓN}

\section{Identificación de los enemigos naturales}

Se encontraron cuatro especies de parasitoides himenópteros en ninfas de la mosca blanca en espiral. Estas especies estuvieron conformadas por Encarsiella noyesi Hayat, E. aleurodici (Girault), Encarsiella prob. nsp. y Encarsia guadeloupae Viggiani todas pertenecientes al orden Hymenoptera, familia Aphelinidae. De estas especies, E. noyesi fue la más abundante. Se conoce muy poco sobre la biología de estas especies; sin embargo, todos son endoparásitos de sus hospederos, donde sólo una larva de los mismos se desarrolla por ninfa de mosca blanca. Observaciones de campo indican que estos insectos parasitan ninfas de la mosca blanca en espiral a partir del estado de "crawler". Las larvas de las avispas consumen las ninfas de mosca blanca y en pocos días emerge la avispa adulta. Los parasitoides adultos posiblemente se alimentan del néctar de las flores de banano recién abiertas (Blanco-Metzler y Laprade, en prensa), de las secreciones azucaradas producidas por las moscas blancas adultas (Lambkin, 1996a) y por el néctar y polen de malezas y otras plantas vecinas. 
Los adultos de los parasitoides son muy pequeños: E. noyesi tiene una longitud de 0,7-0,9 mm; E. aleurodici de 0,8-1,00 mm y Encarsia spp. de 0,5-0,8 mm (Cave, 1995). Estos emergen de sus hospederos por medio de un orificio realizado en el integumento de la momia.

En un recuento sobre proporción de sexos en Encarsiella noyesi (especie más abundante), se encontróuna relación de tres hembras por macho $(\mathrm{n}=36)$. Hanson (1995) informa que en estos insectos, es común la reproducción partenogenética (sin macho), lo que podría explicar dicha proporción.

De los resultados obtenidos, se desprende que de las avispas Encarsia recuperadas, posiblemente sean descendientes de las liberadas por Lambkin en 1996 por lo que se podría decir que estas sí se lograron establecer. Sin embargo, se debe tomar en cuenta que Lambkin no registró cuál fue la especie liberada.

Se encontraron dos especies de ácaros de la familia Phytoseiidae, así como larvas y adultos de Nephaspis sp. y Scymnus sp. (Coleoptera: Coccine1idae) alimentándose de las posturas y ninfas pequeñas de mosca blanca. Posteriormente, observaciones de campo permitieron determinar la presencia de otros depredadores: Polybia sp. (Hymenoptera: Vespidae), Chrysopa sp. (Coleoptera: Chrysopidae) y tres especies de arañas, Plesiometa argyra, Gasteracantha cancriformis, Chrysso sp. Kumashiro et al. (1983) informa de la efectividad en la reducción de las poblaciones de $A$. dispersus en Hawaii por la importación de los enemigos naturales, Nephaspis amnicola y Encarsia ? haitiensis, procedentes de Honduras.

Los ácaros y coccinélidos fueron los depredadores predominantes de A. dispersus y posiblemente los responsables de las reducciones en el número de huevos que pasarían al estado de ninfa. Estos depredadores por lo general se encontraban en áreas donde había poblaciones altas de su hospedero y presentaban poca dispersión hasta que se redujo la población del hospedero. En estudios relaizados sobre la composición taxonómica de los depredadores de las moscas blancas, Gerling (1990) considera que los coccinélidos son los depredadores prevalecientes en climas cálidos donde conviven la plaga y sus depredadores durante todo el año en espacios abiertos, mientras que el grupo de los heteróptoros prevalece bajo condiciones de invernadero. Sin embargo, otro estudio sobre los depredadores de las moscas blancas Bemisia tabaci (Gennadius) y Parabemisia myricae (Kuwana) señalan a los ácaros como un posible componente importante de la mayoría de los complejos de enemigos naturales de las moscas blancas (Gerling 1990).

La presencia de arañas en las plantaciones de banano fue bastante frecuente, tanto en la superficie de las hojas como entre plantas u hojas de banano unidas por medio de telarañas. En la mayoría de los sistemas de producción, la composición de las especies de arañas y su abundancia relativa es poco conocida así como el papel que juegan en el control de plagas en los cultivos. Algunas posibles razones para esto, es que el canibalismo se da principalmente en las noches y no se observa el efecto sobre las plagas. Las arañas poseen ciclos de vida largos, con respecto a las especies plaga y hay pocas personas entrenadas en la identificación de las mismas. Sin embargo, estudios recientes en plagas de la uva (Costello y Daane, 1995), algodón (Bastidas et al., 1994), aguacate (Mansour et al., 1985) resaltan el papel que estos artrópodos han tenido en la regulación de plagas de diversos cultivos.

Vargas (comunicación personal) ${ }^{1}$ identificó al hongo que estaba sobre las ninfas como Niorospora sphaerica e indica que no existen informes donde se clasifique como entomopatógeno. Sin embargo, se requieren más estudios a nivel de laboratorio, conducentes a verificar la relación con el patógeno (postulados de Koch), ya que las observaciones de campo mostraron que las ninfas que tenían este hongo estaban muertas.

\section{Abundancia poblacional}

Se encontraron diferencias altamente significativas para el número de ninfas parasitadas $(\mathrm{F}=39,42 ; \mathrm{gl}=9$; $\mathrm{P}<0,0001)$ entre fincas. El número de ninfas parasitadas fue mayor en la finca AGRODISA $(10,05)$, seguida por SEMDEL $(4,13)$ y Anabel $(3,48)$, mientras que para las otras fincas, las medias oscilaron entre 2,77 y 0,35 . Se registró la menor población de parasitoides en Zent (Cuadro 1).

La diferencia poblacional de parasitoides entre fincas pudo deberse a diferencias poblacionales del hospedero, a diferencias de manejo de la mosca blanca en cada finca, y a condiciones climáticas especialmente precipitación. Dowdesdell (1966) considera a las condiciones climáticas como el factor más evidente que afecta la población de un insecto.

El uso de enemigos naturales contribuye a la conservación del medio ambiente al lograr una disminución

\footnotetext{
1 Vargas, E. 1997. Entomopatógenos de mosca blanca. San José, Universidad de Costa Rica.
} 
Cuadro 1. Promedio de ninfas parasitadas de Aleurodicus dispersus en diez fincas bananeras del cantón de Matina, Limón, 1997-1998.

\begin{tabular}{lcc}
\hline \multirow{2}{*}{ Finca } & \multicolumn{2}{c}{ Ninfas parasitadas } \\
& Medias* & N \\
\hline AGRODISA & $10,05 \mathrm{a}$ & 153 \\
SEMDEL & $4,13 \mathrm{~b}$ & 153 \\
Anabel & $3,48 \mathrm{~b}$ & 153 \\
La Flor & $2,77 \mathrm{c}$ & 153 \\
Hacienda Río Palacios & $2,46 \mathrm{c}$ & 153 \\
PROBANA & $1,92 \mathrm{~cd}$ & 153 \\
Lagartos & $1,52 \mathrm{~d}$ & 153 \\
Rinos & $0,80 \mathrm{de}$ & 153 \\
El Esfuerzo & $0,76 \mathrm{e}$ & 153 \\
Zent & $0,35 \mathrm{e}$ & 153 \\
\hline
\end{tabular}

* Medias seguidas por la misma letra no son significativas según la prueba de Duncan al 0,05. Datos transformados por $\sqrt{X}+0,5$.

en el número de aplicaciones de insecticidas para controlar una plaga. Debido a que se encontró gran variedad de fauna benéfica y que $A$. dispersus no se considera una plaga de importancia económica, es necesario reducir los daños al ambiente (uso más racional de agroquímicos) con el fin de evitar que se rompa el balance entre la mosca blanca y sus enemigos naturales. El hecho de que ninguno de los enemigos naturales encontrados es específico para $A$. dispersus, no se recomienda el control biológico, como única táctica en el manejo de la mosca blanca, sino que la estrategia se debería basar en un diseño de manejo integrado de plagas (MIP).

\section{AGRADECIMIENTO}

Los autores agradecen a los Drs. Paul Hanson y Greg Evans por la identificación de los parasitoides; al Dr. William Eberhard por la identificación de las arañas y a la Ing. Ruth León por facilitar la colección de referencia de insectos en la identificación de los coccinélidos. Se agradece a CORBANA por el apoyo durante el desarrollo de la investigación.

\section{LITERATURA CITADA}

BASTIDAS, H.; MURILLO, A; PANTOJA, A; ZULUAGA, J.I.; GUTIÉRREZ, Y. 1994. Reconocimiento de arañas en algodonero en el Valle del Cauca. Revista MIP No. 32:33-35.
BLANCO-METZLER, H.; LAPRADE, S. 1998. La mosca blanca en espiral Aleurodicus dispersus Russell (Homoptera: Aleyrodidae) en banano. San José, Universidad de Costa Rica. 59 p.

BLANCO-METZLER, H.; LAPRADE, S. En prensa. Hospederos alternos de la mosca blanca en espiral Aleurodicus dispersus Russell (Homoptera: Aleyrodidae) enbanano. CORBANA.

CAVE, R. 1995. Manual para el reconocimiento de parasitoides de plagas agrícolas en América Central. Honduras, El Zamorano, 202 p.

COSTELlO, M.; DAANE, K. 1995. Spider (Araneae) species composition and seasonal abundance in San Joaquin Valley grape vineyards. Environmental Entomology 24(4):823-831.

DOWDESWELL, W.H. 1966. Ecología animal. Madrid, Es paña, Editorial Alhambra. p. 33-87.

HANSON, P. 1995. Aphelinidae. In Hanson y Gauld (Eds). The Hymenoptera of Costa Rica. Oxford University Press. p. 282-289.

KUMASHIRO, B.R.; LAI, P.Y.; FUNASAKI, G.Y.; TERAMOTO, K.K. 1983. Efficacy of Nephaspis amnicola and Encarsia ?haitiensis in controlling Aleurodicus dispersus in Hawaii. Proceedings Hawaiian Entomological Society 24(2-3):261-267.

LAMBKIN, T.A 1996A. Pest status and control of spiralling whitefly Aleurodicus dispersus Russell (Homoptera: Aleyrodidae) in Queensland, Australia. Queensland, Australia, Department of Primary Industries. 58 p.

LAMBKIN, T.A 1996b. Status and control of spiralling whitefly Aleurodicus dispersus Russell in bananas in Costa Rica, Central America. Queensland, Australia, Department of Primary Industries. $16 \mathrm{p}$.

LAPRADE, S.; BLANCO-METZLER, H. En prensa. Efecto del contenido de fumagina, Capnodium sp.? en el rendimiento del banano. CORBANA.

LAPRADE, S.; CERDAS, V. 1997. Manejo de mosca blanca Aleurodicus dispersus Russell (Homoptera: Aleyrodidae) en banano (Musa AAA) utilizando insecticidas y aceite. CORBANA 22(48):89-94.

MANSOUR, E; WYSOKI, M.; WHITCOMB, W.H. 1985. Spiders inhabiting avocado orchards and their role as natural enemies of Boarmia selenaria Schiff. (Lepidoptera: Geometridae) larvae in Israel. Ecology et Applicata 6(4):315-321.

WATERHOUSE, D.E; NORRIS, K.R. 1989. Biological control: Pacific Prospects. Supplement 1. ACIAR Monograph No. 12. p. 12-23. 